\title{
Clinical Characteristics of Patients with Tetralogy of Fallot who Underwent an Invasive Procedure for Arrhythmias
}

\author{
Barbara Adelmann de Lima ${ }^{1, *}$, Antonio Carlos Gallo da Silva², Marco Aurélio Lumertz Saffi², \\ Clóvis Fröemming Junior ${ }^{4}$, Gabriela Castilhos ${ }^{4}$, Marcelo Lapa Kruse ${ }^{4}$, Gustavo Glotz de \\ Lima $^{2,4}$, Tiago Luiz Luz Leiria ${ }^{4}$
}

\section{ORCID ID}

Lima BA (D) https://orcid.org/0000-0002-3531-4128

Silva ACG (D) https://orcid.org/0000-0001-8614-6841

Saffi MAL (D) https://orcid.org/0000-0001-8232-3310

Fröemming Junior C (D) https://orcid.org/0000-0002-4770-0050

\author{
Castilhos G (D) https://orcid.org/0000-0001-6738-6065 \\ Kruse ML (D) https://orcid.org/0000-0002-2429-4491 \\ Lima GG (D) https://orcid.org/0000-0003-0097-5206 \\ Leiria TLL (D) https://orcid.org/0000-0002-3905-102X
}

\begin{abstract}
Introduction: Tetralogy of Fallot (TOF) is a cyanotic congenital heart disease that has an incidence of sudden cardiac death of $0.2 \%$ per year, being arrhythmias the main cause of its occurrence. Objective: To compare characteristics of TOF patients referred for electrophysiological study (EPS) against those that were not (No-EPS). Method: Retrospective cohort with 215 patients (57.2\% men; age $=29 \pm 4$ ) with corrected TOF (median of three years, ranging from 0.33 to 51) that underwent EPS between 2009-2020. The primary outcome was composed of death, implantable cardiac defibrillator (ICD) requirement and hospitalization. Results: Pre-syncope $($ EPS $=4.7 \%$, No-EPS = 0\%; $p=0.004)$, syncope $($ EPS $=7.1 \%$, No-EPS $=1.7 \% ; p=0.056)$ and palpitations $(E P S=31 \%$, No-EPS = 5.8\%; $p<0.001$ ) were symptoms that justified electrophysiological investigation. ICD was implanted in 24\% of EPS and 0.6\% of No-EPS $(p=0.001)$. Twenty-six percent of the EPS group presented non-sustained ventricular tachycardia, while 0\% in No-EPS ( $p=0.012)$. The EPS group had more atrial fibrillation or atrial Flutter (35.7\% vs. 6.9\%; $p<0.001$ ). The EPS patients had a wider QRS duration than the no-EPS group (171.12 \pm 29.52 ms vs. $147 \pm 29.77$ ms; $p<0.001$ ). Also, 26.2\% of EPS performed ablation to correct macroreentrant atrial tachycardias. The incidence of primary outcome (death + ICD requirement + hospitalization) was higher in patients in the EPS group compared to the No-EPS group ( $p=0.001$ ). However, the total of seven deaths occurred during the clinical follow-up, but without differences between the groups (EPS $=4.7 \%$ vs. No-EPS $=2.8 \% ; p=0.480$ ). Conclusion: EPS group had a profile of greater risk, more complex heart disease, and a greater occurrence of the primary outcome when compared to the No-EPS group.
\end{abstract}

KEYWORDS: Tetralogy of Fallot; Cardiac Electrophysiology, Tetralogy of Fallot; Arrhythmias, Cardiac.

1. Pontifícia Universidade Católica do Rio Grande do Sul - Escola de Medicina - Porto Alegre (RS), Brazil.

2. Universidade Federal de Ciências da Saúde de Porto Alegre - Porto Alegre (RS), Brazil.

3. Hospital de Clínicas de Porto Alegre - Porto Alegre (RS), Brazil.

4. Instituto de Cardiologia do Rio Grande do Sul - Porto Alegre (RS), Brazil.

*Corresponding author: barbaraadelmanndelima.med@gmail.com

Received: Apr 22, 2021 | Accepted: Dec 7, 2021 


\section{INTRODUCTION}

Tetralogy of Fallot (TOF) is a cyanotic congenital heart disease with an incidence of one in 3,500 births, accounting for up to one-tenth of all congenital cardiac injuries ${ }^{1,2}$. This pathology comprises four fundamental anatomical abnormalities: ventricular septal defect, pulmonary artery stenosis, hypertrophy of the right ventricle, and extraposition of the aorta over the septum ${ }^{2}$. Before the advent of surgical intervention, about $50 \%$ of patients with TOF died in the first years of life, and it was rare for patients to survive beyond 30 years of age ${ }^{3}$. Currently, about $85 \%$ of patients who have had surgical corrections reach adulthood ${ }^{4}$. Still, over the years, these patients have had an incidence of 0.5 to $6 \%$ of sudden cardiac death ${ }^{5}$. Patients undergoing a surgical correction in the future may have complications such as pulmonary regurgitation, recurrence of pulmonary stenosis, and ventricular arrhythmias ${ }^{2}$.

Approximately $75 \%$ of sudden deaths in patients with corrected TOF are secondary to ventricular arrhythmias ${ }^{6}$. In this context, the patient's age is an aggravating factor for sudden death, since the risk of sustained ventricular tachycardia increases from $0.2 \%$ to $0.5 \%$ per year in operated and followed-up patients after 21 and 30 years old, respectively ${ }^{7,8}$. Some atrial arrhythmias, such as fibrillation and atrial flutter, are associated with a greater chance of death, predisposing to more severe ventricular arrhythmias. ${ }^{9}$. Other mechanisms of sudden death result from anatomical changes that can cause hemodynamic impairment, embolism, myocardial infarction, or rupture of an aneurysm ${ }^{10}$.

The risk factors for sudden cardiac death that are considered in patients with TOF are left systolic ventricular dysfunction, presyncope, syncope, palpitations, QRS duration equals to or greater than $180 \mathrm{~ms}$, non-sustained ventricular tachycardia, and induction of ventricular arrhythmias in an electrophysiological study (EPS) ${ }^{11}$. To mitigate sudden death, the induction of sustained ventricular tachyarrhythmias in EPS becomes an important tool in stratifying patients with these risk factors in the decision to implant a cardiac defibrillator?.

Considering this scenario of late complications after TOF correction, this study aimed to assess the clinical characteristics of patients who underwent EPS, and analyze the clinical outcomes (mortality, hospitalization and implantable cardiac defibrillator - ICD) after the procedure.

\section{METHODS}

This was a retrospective cohort study to assess the differences over time in morbidity and mortality in patients diagnosed with TOF that were referred to EPS. The database used for this study comprised electronic medical records from 2009 to 2020 for all patients who were surgically treated for TOF at the Institute of Cardiology of Rio Grande do Sul, of Fundação Universitária de Cardiologia (IC-FUC). This study was approved by the IC-FUC research ethics committee under case number 1.966.902. All data verified in the profile studied followed the premises of the Declaration of Helsinki and the Nuremberg Code, respecting the Research Standards Involving Human Beings (Resolution No. 466/2012), of the National Health Council.

All patients who underwent surgical correction for TOF were eligible for the study. The clinical follow-up occurred through regular consultations in the congenital heart disease laboratory, and the updating of medical records was performed by the responsible medical team. Data collection occurred by reviewing electronic and physical records and by analyzing electrophysiological reports (between 2009-2020) for patients who underwent the arrhythmia investigation procedure. Thus, patients with TOF were divided into two distinct groups. The first group consists of patients who underwent the EPS in adulthood (EPS), which were compared with patients who did not perform the EPS in adulthood (No-EPS).

Demographic data (age and gender), clinical history (age at TOF surgery, presyncope, syncope, palpitations, implantation of biological pulmonary valve, implantation of cardioverter-defibrillator, coronary anomaly, pulmonary valve agenesis, and the number of hospitalizations), electrocardiographic characteristics (rhythm, duration of the QRS complex, nonsustained ventricular tachycardia and atrial fibrillation/flutter), associated comorbidities (hypertension, diabetes, obesity, hypothyroidism, interatrial communication, and dyslipidemia) were evaluated by a cardiologist and reviewed by an electrophysiologist. Death, ICD, and the number of hospitalizations were considered as the primary clinical outcomes for 
investigating the effects of EPS. Hospitalizations to perform the EPS were not considered as an outcome. We defined the primary outcome as a composite of death + ICD requirement + hospitalization, which was created to assess the overall effect of invasive assessment of arrhythmias in these patients.

\section{Statistical analysis}

Statistical analyses were processed using Statistical Package for the Social Sciences (SPSS) software, version 16.0 (IBM Inc., Chicago, IL, United States of America). Absolute frequencies and percentages were used to characterize the sample. Continuous variables were described as means and standard deviations, or medians and interquartile ranges for non-normally distributed data. Continuous variables were compared using the t-test for independent samples or nonparametric test as indicated. Categorical variables were compared using the $₫ 2$ test. We considered $p \leq 0.05$ statistically significant. The analysis of event-free survival was performed using the Kaplan-Meier method, which was presented as a percentage of cumulative survival, and comparisons using the log-rank test.

To find risk stratifies for the combined outcome (death/ICD/hospitalization), we performed a univariate analysis followed by multivariate analysis to identify the variables of interest. We considered $\mathrm{p} \leq 0.05$ statistically significant. Analysis was performed using the Cox regression. The missing data in the medical records were treated using the multiple imputation method by SPSS. This way, multiple imputations produce appropriate results, even in the presence of small sample size or a large number of missing data ${ }^{8}$.

\section{RESULTS}

Two hundred and fifteen patients were identified with TOF in the database, 42 patients (20\%) underwent EPS, and the remaining 173 patients (80\%) did not (No-EPS) (Fig. 1).

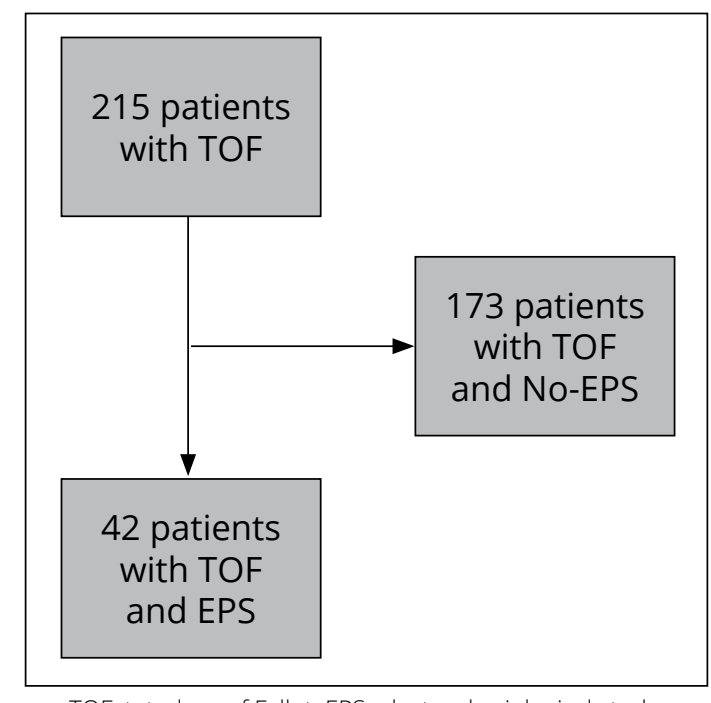

TOF: tetralogy of Fallot; EPS: electrophysiological study.

Figure 1. Study flowchart.

The EPS indications were supraventricular extrasystoles in eight cases, atrial flutter in one case, second degree atrialventricular block in one case, and history of ventricular tachycardia in two cases. The main clinical and demographic characteristics of the patients are shown in Table 1. Between the groups, the systolic pressure in the right ventricle was the same (EPS, $44.92 \pm 25.22 \mathrm{mmHg}$ vs. No-EPS, $37.65 \pm 13.42 \mathrm{mmHg}$; $=0.331$ ), while the group submitted to the invasive procedure had greater QRS width (EPS, $171.12 \pm 29.52 \mathrm{~ms}$ vs. No-EPS, $147 \pm 29.77 \mathrm{~ms} ; \mathrm{p}<0.001$ ). Additionally, in the EPS group, $26 \%$ of the patients had non-sustained ventricular tachycardia [9/42 (21.4\%) vs. 0/173 (0\%); p = 0.012), and 35.7\% had a higher AF/Flutter ratio [15/42 (35.7\%) vs. 12/173 (6.9\%); $\mathrm{p}<0.001)]$ in comparison with the non-EPS group (Table 1). 
Table 1. Clinical differences between patients with surgically corrected TOF who performed the EPS and who did not perform it.

\begin{tabular}{|c|c|c|c|}
\hline Characteristics & EPS $(n=42)$ & No-EPS $(n=173)$ & p-value* \\
\hline \multicolumn{4}{|l|}{ Sociodemographic } \\
\hline Age (years) \pm mean $($ sd) & $36.9 \pm 11.1$ & $30.1 \pm 8.4$ & 0.001 \\
\hline Male (\%) & $24(57.1 \%)$ & $99(57.2 \%)$ & 0.819 \\
\hline Age TOF Sx (years) \pm mean (sd) & $4.5 \pm 4.7$ & $4.3 \pm 5.7$ & 0.612 \\
\hline \multicolumn{4}{|l|}{ Comorbidities } \\
\hline Hypertension & $2(4.8 \%)$ & $4(2.3 \%)$ & 0.338 \\
\hline Diabetes & - & $2(1.1 \%)$ & 0.500 \\
\hline IAC & $3(7.1 \%)$ & $10(5.8 \%)$ & 0.246 \\
\hline Obesity & $3(7.1 \%)$ & $8(4.6 \%)$ & 0.430 \\
\hline Hypothyroidism & $1(2.4 \%)$ & $6(3.5 \%)$ & 0.775 \\
\hline Dyslipidemia & - & $5(2.9 \%)$ & 0.283 \\
\hline \multicolumn{4}{|l|}{ Echocardiogram } \\
\hline Biological PV & $12(28.6 \%)$ & $21(12.1 \%)$ & 0.003 \\
\hline Coronary anomaly & $4(9.5 \%)$ & $3(1.7 \%)$ & 0.005 \\
\hline Agenesis PV & $4(9.5 \%)$ & $1(0.6 \%)$ & 0.001 \\
\hline \multicolumn{4}{|l|}{ Electrocardiogram } \\
\hline Sinus rhythm & $38(90.5 \%)$ & $165(95.4 \%)$ & 0.480 \\
\hline NSVT & $9(21.4 \%)$ & - & 0.012 \\
\hline AF/Flutter & $15(35.7 \%)$ & $12(6.9 \%)$ & 0.001 \\
\hline QRS (ms) \pm mean (sd) & $171 \pm 30$ & $147 \pm 30$ & 0.001 \\
\hline \multicolumn{4}{|l|}{ Clinical manifestation } \\
\hline Pre-syncope & $2(4.8 \%)$ & - & 0.004 \\
\hline Syncope & $3(7.1 \%)$ & $3(1.7 \%)$ & 0.056 \\
\hline Palpitation & $13(30.9 \%)$ & $10(5.8 \%)$ & 0.001 \\
\hline
\end{tabular}

TOF: tetralogy of Fallot; EPS: electrophysiological study; sd: standard deviation; Sx: surgery; IAC: interatrial communication; PV: pulmonary valve; coronary An.: Coronary Anomaly; NSVT: non-sustained ventricular tachycardia; AF: atrial fibrillation; *p-value expresses the difference of the Student's T-test for continuous variables (age, age at TOF Sx, QRS duration) and the $x 2$ for categorical variables (male, comorbidities, echocardiogram findings, and sinus rhythm). The statistical significance adopted was 5\%.

There was an increase in atrial flutter and atrial fibrillation in the group that performed the EPS [15/42 (35.7\%) vs. 12/173 (6.9\%)]. Pre-syncope [2/42 (4.8\%) vs. 0/173] and palpitations [13/42 (30.9\%) vs. 10/173 (5.8\%)] represented the majority of the most incidents, with the tendency to increase in the proportion of syncope, although not statistically confirmed.

During the electrophysiological study, 12 of the 42 patients had an induced VT, and other 12 ones had an SVT inducible (Table 2). The patients with induced VT already had high incidence of ventricular extra systoles (23\%) as a characteristic before the EPS was conducted. The total of 11 patients were submitted to catheter ablation, 10 patients underwent successful atrial flutter catheter ablation (26\%), and the accessory pathway was successfully ablated in one patient.

Table 2. EPS results.

\begin{tabular}{lc}
\multicolumn{1}{c}{ EPS Results } & $\mathbf{N}=\mathbf{4 2}$ \\
\hline Inducible arrhythmias & $\mathbf{2 1 ( 5 0 \% )}$ \\
$\cdot$ No arrhythmia inducible & $30(71.4 \%)$ \\
$\cdot$ VT & $12(28.6 \%)$ \\
$\cdot$ SVT & $12(28.6 \%)$ \\
Catheter ablation & $11(23.8 \%)$ \\
$\cdot$ Atrial flutter & $10(23.8 \%)$ \\
- Accessory pathway & $1(2.4 \%)$ \\
\hline
\end{tabular}

3 patients had an induced VT as well as an SVT inducible. EPS: electrophysiological study; VT induction: induction of ventricular tachycardia; SVT induction: induction of supraventricular tachycardia. 
The incidence of combined outcomes (death, hospitalizations, ICD implantation) was higher in EPS patients compared to the No-EPS [18 (42.9\%) vs. 25 (14.4\%); p = 0.001] (Table 3). There were no differences between the groups regarding cumulative occurrences of death [2 (4.8\%) vs. 5 (2.9\%); p = 0.480]. However, hospitalizations [9 (21.4\%) vs. 19 (11\%); $\mathrm{p}=0.012)$ ] and of ICD implantation [10 (23.8\%) vs. 1 (0.6\%); $<$ 0.001] were more significantly observed in the EPS group (Table 3). In a cumulative analysis, there was a higher incidence of ICDs implantation in the EPS group (p = 0.0293; Fig. 2).

Table 3. Difference in clinical outcomes presented by patients with surgically corrected TOF, compared between groups that performed the EPS and those who did not perform it.

\begin{tabular}{lccc}
\hline \multicolumn{1}{c}{ Characteristics } & EPS $(\mathbf{n}=42)$ & No-EPS $(\mathbf{n}=173)$ & p-value* \\
\hline Primary outcome & & & 0.001 \\
\hline Combined & $18(42.9 \%)$ & $25(14.4 \%)$ & \\
\hline Secondary outcome & & & 0.480 \\
\hline Death (\%) & $2(4.8 \%)$ & $5(2.9 \%)$ & 0.001 \\
ICD (\%) & $10(23.8 \%)$ & $1(0.6 \%)$ & 0.012 \\
Hospitalization (\%) & $9(21.4 \%)$ & $19(11 \%)$ & \\
\hline
\end{tabular}

TOF: tetralogy of Fallot; EPS: electrophysiological study; ICD: implantable cardiac defibrillator; ${ }^{\star} \mathrm{p}$-value expresses the result of the $x 2$ test for categorical variables (death, ICD, and hospitalization). The statistical significance adopted was 5\%.

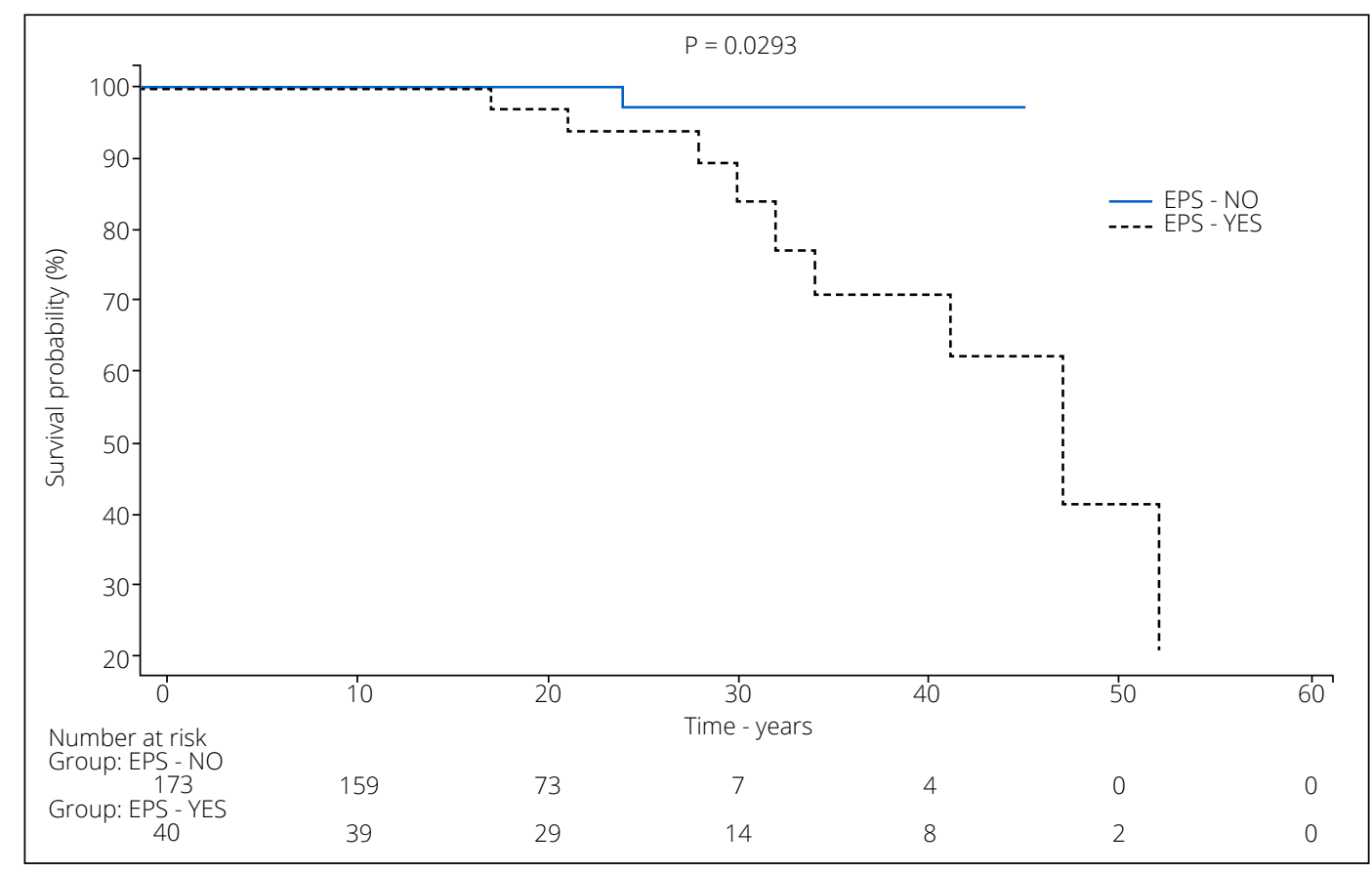

TOF: tetralogy of Fallot; *Log-rank test: $p=0.0293$; EPS - NO: patients who did not perform an electrophysiological study;

EPS - YES: patients who underwent an electrophysiological study.

Figure 2. Cumulative survival of cardioverter-defibrillator implantation in patients with surgically corrected TOF*.

Univariate associations between combined outcome and risk stratifies are presented in Table 4. Age at last appointment (odds ratio $-\mathrm{OR}=0.80$ [95\% confidence interval - 95\%CI 0.74-0.87]), AF/Flutter (OR = 2.16 [95\%CI 1.12-4.16]) and $\mathrm{QRS}$ duration $(\mathrm{OR}=1.012$; [95\% CI 1.01-1.02] were the variables that best justified the combined outcome separately. Three stratifies emerged as significantly associated with a combined outcome in the multivariable model (Table 5). Age at last appointment $(\mathrm{OR}=0.77$ [95\%CI 0.70-0.84]), as a protection factor and AF/Flutter $(\mathrm{OR}=4.55[95 \% \mathrm{CI} 1.96-10.58])$ and $\mathrm{QRS}$ duration $(\mathrm{OR}=1.02$ [95\% CI 1.01-1.03] as negative risk predictors were the variables that best justified the combined outcome. 
Table 4. Univariate analysis.

\begin{tabular}{lccc}
\hline & Odds ratio & 95\%Cl & p-value \\
\hline EPS & 1.05 & $(0.54-2.05)$ & 0.89 \\
Age at last appointment & 0.80 & $(0.74-0.87)$ & $<0.01$ \\
Sex & 0.74 & $(0.40-1.39)$ & 0.35 \\
NSVT & 0.77 & $(0.27-2.20)$ & 0.63 \\
AF/Flutter & 2.16 & $(1.12-4.16)$ & $<0.01$ \\
QRS duration & 1.012 & $(1.01-1.02)$ & $<0.01$ \\
\hline
\end{tabular}

95\%Cl: 95\% confidence interval; EPS: electrophysiological study; NSVT: non-sustained ventricular tachycardia; AF: atrial fibrillation.

Table 5. Multivariate analysis.

\begin{tabular}{lccc}
\hline & Odds ratio & $95 \% \mathrm{Cl}$ & p-value \\
\hline EPS & 0.72 & $(0.29-1.76)$ & 0.48 \\
Age at last appointment & 0.77 & $(0.70-0.84)$ & $<0.01$ \\
AF/Flutter & 4.55 & $(1.96-10.58)$ & $<0.01$ \\
QRS duration & 1.02 & $(1.01-1.03)$ & $<0.01$ \\
\hline
\end{tabular}

95\%Cl: 95\% confidence interval; EPS: electrophysiological study; AF: atrial fibrillation.

\section{DISCUSSION}

In our study, we compared patients with surgically corrected TOF who underwent EPS with those who did not undergo invasive examination. We found a predominance of changes in cardiovascular function, increase in atrial flutter, and atrial fibrillation in the group that performed the EPS. Among the clinical manifestations that are indicative of the EPS, pre-syncope and palpitations represented the majority of the most incidents, with tendency to increase in the proportion of syncope, although not statistically confirmed. Among arrhythmias, the most prevalent in patients with TOF was nonsustained ventricular tachycardia. Also, we observed that the ICD is greater in patients undergoing invasive examination, which is compatible with the fact that these patients have more complex heart disease than the other patients studied. Such findings are in line with the objective proposed by the study, which was to evaluate the clinical characteristics of patients with a congenital malformation (TOF) even if surgically corrected.

Patients with surgically corrected TOF may experience severe cardiac arrhythmias that become more frequent with advancing age. Nowadays, it is known that ventricular arrhythmias are the most common cause of mortality in patients with corrected $\mathrm{TOF}^{12}$, which is compatible with the seven deaths that we found in the evaluated database. The fact of having a biological pulmonary valve and coronary anomaly may be responsible for a higher density of cardiac arrhythmias due to myocardial ischemia for the abnormal coronary and a greater number of interventions in the case of a biological pulmonary valve.

Although there is no difference between the groups studied, the proportion of reasons for death is different. The two deaths in the group that underwent intervention were caused by sudden death and hemorrhagic shock, while the deaths in the group without intervention were due to a hemorrhagic shock event and four occurrences of cardiogenic shock. Furthermore, the death of these patients may also be related to left systolic ventricular dysfunction, presyncope, syncope, palpitations, QRS duration equal to or greater than $180 \mathrm{~ms}$, non-sustained ventricular tachycardia, and induction of ventricular arrhythmias in $\mathrm{EPS}^{12}$. These findings reinforce the evidence that patients with corrected TOF have higher risk of lethal cardiovascular events.

Khairy et al. ${ }^{11}$ demonstrated that the risk factors for sudden death were advanced age in repair surgery, ventricular fibrosis, impaired hemodynamics, and electrophysiological manifestations, representing events that are seldomly found in our database (Table 1). However, based on our multivariate and univariate analyses, we were able to identify that the presence of AF/Flutter was the characteristic that most significantly affected the primary outcome (death + ICD requirement + hospitalization) (Tables 4 and 5). This is due to the risks that involve arrhythmias, such as sudden cardiac death. QRS duration also significantly affected the 
primary outcome, because a larger QRS duration can cause arrhythmias, and these can also lead to sudden cardiac death. Thus, it can be said that EPS is important in the stratification of patients at risk for Sudden Cardiac Death (SCD) and ICD implantation.

In the context of clinical findings, within the scope of conducting the EPS, patients with supraventricular tachyarrhythmia have more symptoms and greater structural and physical repercussions of the disease ${ }^{13}$. This statement is corroborated by our results since the group of patients undergoing invasive treatment had a higher proportion of presyncope, syncope, and palpitations. It is based on these findings that we reinforce the statement that patients with TOF are the ones that receive the most ICD among all others with any congenital heart disease ${ }^{13}$. More specifically, among the 215 patients who participated in our cohort, 11 patients received ICD (6.3\%), 10 of which underwent EPS, representing 23.8\% of these patients (Table 3). Highlighting, once again, the complexity of cardiomyopathy in patients with TOF corrected by surgery, especially in the group that performed the EPS. It is also important to notice that no patient with an ICD implant died.

It is also noteworthy that the patients who underwent the EPS had a higher mortality rate and a higher hospitalization rate than the group that did not undergo intervention (Table 3). Despite the possibility of a higher mortality rate in patients who underwent EPS being related to a more complex structural heart disease, we cannot corroborate this finding based on the data that we have presented. The occurrence of only seven deaths limits our inferential capacity and the internal validity of the finding. Also, there is no statistical support to state that there is a difference in the number of deaths between the groups studied. The higher prevalence of hospitalization in the group that performed the EPS intervention compared to the group that did not reinforce the statement that these patients have more complex cardiomyopathy and, consequently, experienced worse clinical repercussions.

The main limitations of this study are related to the study design, as it is a retrospective and observational cohort. There is still data that is missing, despite the correction by the multiple pairwise methods. In addition, the use of data restricted to IC-FUC patients restricts our ability to externally generalize with other hospital centers. We also identified as a selection bias the inclusion of patients without discrimination due to the surgical technique performed and the degree of the congenital anomaly presented by the patients. However, this study described what happens in the clinical practice of a referral hospital specialized in cardiology.

\section{CONCLUSION}

Based on the findings presented in this article, we can suggest that EPS is another tool to stratify the severity of patients with surgically corrected TOF, while also identifying patients at higher risk because the EPS induces non-sustained ventricular tachycardia in patients without previous evidence of arrhythmia for whom ICD was indicated.

Based on our multivariate and univariate analyses, we were able to identify that the presence of AF/Flutter was the characteristic that most significantly affected the primary outcome. This is due to the risks that involve arrhythmias, such as sudden cardiac death. QRS duration also significantly affected the primary outcome, because a bigger QRS duration can cause arrhythmias, and these can lead to sudden cardiac death. In contrast, the age at the last appointment is a protecting factor, as it reflects a patient profile that is more cautious with their medical condition.

It was possible to observe that patients with surgically corrected TOF referred to EPS had more complex heart disease when compared to those who did not undergo the procedure. This implies that the clinical conditions of presyncope, syncope, and hospitalization are good predictors of the TOF prognosis. Additionally, the EPS was a diagnostic tool widely used for the decision to implant an ICD, when confronted with electrophysiological abnormalities presented by patients.

\section{AUTHOR'S CONTRIBUTION}

Conceptualization: Leiria TLL, Kruse ML, Lima GG; Formal Analysis: Lima BA, Gallo AC; Writing - original draft: Leiria TLL, Kruse ML, Lima GG , Lima BA, Gallo AC, Saffi MAL; Junior CF, Castilhos G; Writing - review \& editing: Lima BA, Gallo AC, Saffi MAL. 


\section{DATA AVAILABILITY STATEMENT}

Raw data were generated at Institute of Cardiology of Rio Grande do Sul, of Fundação Universitária de Cardiologia. The data that support the findings of this study are available on request from the corresponding author.

\section{FUNDING}

Fundo de Apoio do Instituto de Cardiologia para Ciência e Cultura

Fundação de Amparo à Pesquisa do Estado do Rio Grande do Sul

https://doi.org/10.13039/501100004263

\section{ACKNOWLEDGMENTS}

The authors would like to thank the IC-FUC for providing all the infrastructure necessary for the research. The authors also thank the Scientific Department of IC-FUC, in particular Madalena Cristina and Fernanda Poester, for their excellent office support.

\section{CONFLICT OF INTERESTS}

The authors declare no competing interests.

\section{REFERENCES}

1. Villafañe J, Feinstein JA, J Jenkins KJ, Vincent RN, P Walsh EP, Dubin AM, et al. Hot topics in tetralogy of Fallot. J Am Coll Cardiol. 2013;62(23):2155-66. https://doi.org/10.1016/j.jacc.2013.07.100

2. Apitz C, Webb GD, Redington AN. Tetralogy of Fallot. Lancet. 2009;374(9699):1462-71. https://doi.org/10.1016/s01406736(09)60657-7

3. Bertranou EG, Blackstone EH, Hazelrig JB, Turner ME, Kirklin JW. Life expectancy without surgery in tetralogy of Fallot. Am J Cardiol. 1978;42(3):458-66. https://doi.org/10.1016/0002-9149(78)90941-4

4. Papagiannis JK. Postoperative arrhythmias in tetralogy of Fallot. Hell J Cardiol [Internet]. 2005 [cited on March 21, 2021];46(6):402-7. Available from: https://www.hellenicjcardiol.org/archive/full_text/2005/6/2005_6_402.pdf

5. Van Doorn C. The unnatural history of tetralogy of Fallot: surgical repair is not as definitive as previously thought. Heart. 2002;88(5):447-8. http://dx.doi.org/10.1136/heart.88.5.447

6. Blomström-Lundqvist C, Scheinman MM, Aliot EM, Alpert JS, Calkins H, Camm AJ, et al. ACC/AHA/ESC guidelines for the management of patients with supraventricular arrhythmias--executive summary: a report of the American College of Cardiology/American Heart Association Task Force on Practice Guidelines and the European Society of Cardiology Committee for Practice Guidelines (Writing Committee to Develop Guidelines for the Management of Patients With Supraventricular Arrhythmias). Circulation. 2003;108(15):1871-909. https://doi.org/10.1161/01.cir.0000091380.04100.84

7. Gatzoulis MA, Balaji S, Webber SA, Siu SC, Hokanson JS, Poile C, et al. Risk factors for arrhythmia and sudden cardiac death late after repair of tetralogy of Fallot: a multicentre study. Lancet. 2000;356(9234):975-81. https://doi.org/10.1016/s0140-6736(00)02714-8 
8. Khairy P, Aboulhosn J, Gurvitz MZ, Opotowsky AR, Mongeon F-P, Kay J, et al. Arrhythmia burden in adults with surgically repaired tetralogy of Fallot: a multi-institutional study. Circulation. 2010;122(9):868-75. https://doi.org/10.1161/circulationaha.109.928481

9. Oliveira GS, Oliveira FS, Osório APS, Medeiros AK, Sant'anna JRM, Leiria TLL. Late outcome and predictors of adverse events related to the implantation of a permanent pacemaker in patients with isolated congenital atrioventricular block. Pediatr Cardiol. 2021;37(7):1319-27. https://doi.org/10.1007/s00246-016-1437-0

10. Maury P, Sacher F, Rollin A, Mondoly P, Duparc A, Zeppenfeld K, et al. Ventricular arrhythmias and sudden death in tetralogy of Fallot. Arch Cardiovasc Dis. 2017;110(5):354-362. https://doi.org/10.1016/j.acvd.2016.12.006

11. Khairy P, Harris L, Landzberg MJ, Viswanathan S, Barlow A, Gatzoulis MA, et al. Implantable cardioverter-defibrillators in tetralogy of Fallot. Circulation. 2008;117(3):363-70. https://doi.org/10.1161/circulationaha.107.726372

12. Page RL, José JA, Caldwell MA, Calkins H, Conti JB, Deal BJ, et al. 2015 ACC/AHA/HRS Guideline for the Management of Adult Patients with Supraventricular Tachycardia: A Report of the American College of Cardiology/American Heart Association Task Force on Clinical Practice Guidelines and the Heart Rhythm Society. J Am Coll Cardiol. 2016;67(13):e27-e115. https://doi.org/10.1016/j. jacc.2015.08.856

13. Khairy P. Arrhythmias in adults with congenital heart disease: what the practicing cardiologist needs to know. Can J Cardiol. 2019;35(12):1698-707. https://doi.org/10.1016/j.cjca.2019.07.009 